\title{
Equation of state of classical Coulomb plasma mixtures
}

\author{
Alexander Y. Potekhin $*$ and Gilles Chabriei甲 \\ Ecole Normale Supérieure de Lyon, CRAL (UMR CNRS No. 5574), 69364 Lyon Cedex 07, France \\ Forrest J. Rogers \\ Lawrence Livermore National Laboratory, P. O. Box 808, Livermore, CA 94550, USA
}

(Dated: November 4, 2018)

\begin{abstract}
We develop analytic approximations of thermodynamic functions of fully ionized nonideal electronion plasma mixtures. In the regime of strong Coulomb coupling, we use our previously developed analytic approximations for the free energy of one-component plasmas with rigid and polarizable electron background and apply the linear mixing rule (LMR). Other thermodynamic functions are obtained through analytic derivation of this free energy. In order to obtain an analytic approximation for the intermediate coupling and transition to the Debye-Hückel limit, we perform hypernettedchain calculations of the free energy, internal energy, and pressure for mixtures of different ion species and introduce a correction to the LMR, which allows a smooth transition from strong to weak Coulomb coupling in agreement with the numerical results.
\end{abstract}

PACS numbers: 52.25.Kn, 05.70.Ce, 52.27.Gr

\section{INTRODUCTION}

We study the equation of state (EOS) of fully ionized nonideal electron-ion plasmas (EIP). In a previous work [1, 2], hypernetted chain (HNC) calculations were performed and analytic formulae were proposed for EOS calculations of EIP containing a single ion species. For mixtures of different ion species, the EOS was calculated using the linear mixing rule (LMR), whose high accuracy at $\Gamma>1$ was previously confirmed in a number of studies $[3,4,5,6,6,7]$. However, the LMR is inaccurate for weakly coupled plasmas. Some consequences of its violation were studied by Nadyozhin and Yudin [8], who showed that the differences between the linear and nonlinear mixing at moderate Coulomb coupling $(0.1 \lesssim \Gamma \lesssim 1)$ can shift the nuclear statistical equilibrium at the final stage of a stellar gravitational collapse.

In this paper, we perform $\mathrm{HNC}$ calculations of the free energy, internal energy, and pressure for mixtures of various kinds of ions in the weak, intermediate, and strong coupling regimes, and suggest an analytic correction to the LMR.

In Sec. II we define the basic plasma parameters. In Sec. [II] we calculate the EOS of ion mixtures and propose an analytic formula for the EOS of multicomponent EIP, applicable at any $\Gamma$ values. The summary is given in Sec. IV.

*Also at the Ioffe Physical-Technical Institute, 194021 St. Petersburg, Russia; Electronic address: palex@astro.ioffe.ru

${ }^{\dagger}$ Electronic address: chabrier@ens-lyon.fr

\section{PLASMA PARAMETERS}

Let $n_{e}$ be the electron number density and $n_{j}$ the number density of ion species $j=1,2, \ldots$, with mass and charge numbers $A_{j}$ and $Z_{j}$, respectively. The total number density of ions is $n_{\text {ion }}=\sum_{j} n_{j}$. The electric neutrality implies $n_{e}=\langle Z\rangle n_{\text {ion }}$. Here and hereafter the brackets $\langle\ldots\rangle$ denote averaging of the type $\langle f\rangle=\sum_{j} x_{j} f_{j}$, where $x_{j} \equiv n_{j} / n_{\text {ion }}$.

The state of a free electron gas is determined by the electron number density $n_{e}$ and temperature $T$. Instead of $n_{e}$ it is convenient to introduce the dimensionless density parameter $r_{s}=a_{e} / a_{0}$, where $a_{e}=\left(\frac{4}{3} \pi n_{e}\right)^{-1 / 3}$ and $a_{0}$ is the Bohr radius.

At stellar densities it is convenient to use, instead of $r_{s}$, the relativity parameter [10] $x_{\text {rel }}=p_{F} / m_{e} c=0.014 r_{s}^{-1}$, where $p_{F}=\hbar\left(3 \pi^{2} n_{e}\right)^{1 / 3}$ is the electron Fermi momentum. The Fermi kinetic energy is $\epsilon_{F}=c \sqrt{\left(m_{e} c\right)^{2}+p_{F}^{2}}-$ $m_{e} c^{2}$, and the Fermi temperature equals $T_{F} \equiv \epsilon_{F} / k_{B}=$ $T_{r}\left(\gamma_{r}-1\right)$, where $T_{r} \equiv m_{e} c^{2} / k_{B}=5.93 \times 10^{9} \mathrm{~K}$, $\gamma_{r} \equiv \sqrt{1+x_{\text {rel }}^{2}}$, and $k_{B}$ is the Boltzmann constant.

The ions are nonrelativistic in most applications. The strength of the Coulomb interaction of ion species $j$ is characterized by the Coulomb coupling parameter,

$$
\Gamma_{j}=\left(Z_{j} e\right)^{2} /\left(a_{j} k_{B} T\right)=\Gamma_{e} Z_{j}^{5 / 3},
$$

where $a_{j}=a_{e} Z_{j}^{1 / 3}$ is the ion sphere radius and $\Gamma_{e} \equiv$ $e^{2} /\left(a_{e} k_{B} T\right)$. In a multicomponent plasma, it is useful to introduce the mean ion-coupling parameter $\Gamma=\Gamma_{e}\left\langle Z^{5 / 3}\right\rangle$ [3]. At a melting temperature $T_{m}$, corresponding to $\Gamma \approx$ 175 (e.g., [2]), the plasma freezes into a Coulomb crystal.

An important scale length is the thermal de Broglie wavelength $\lambda_{j}=\left(2 \pi \hbar^{2} / m_{j} k_{B} T\right)^{1 / 2}$, where $m_{j}$ is the ion mass. The electron thermal length $\lambda_{e}$ is given by the same expression with $m_{j}$ replaced by $m_{e}$.

The quantum effects on ion motion become important at $T \ll T_{p}$, where $T_{p} \equiv \hbar \omega_{p} / k_{B}$ and $\omega_{p}=$ 
$\left(4 \pi e^{2} n_{\text {ion }}\left\langle Z^{2} / m\right\rangle\right)^{1 / 2}$ is the ion plasma frequency.

In this paper we consider only the classical Coulomb liquid, which implies $T \gg T_{p}$ and $T>T_{m}$.

\section{EQUATION OF STATE}

Assuming commutativity of the kinetic and potential operators and separation of the traces of the electronic and ionic parts of the Hamiltonian, the total Helmholtz free energy $F$ can be conveniently written as

$$
F=F_{\text {id }}^{\text {ion }}+F_{\text {id }}^{(e)}+F_{e e}+F_{i i}+F_{i e},
$$

where $F_{\text {id }}^{\text {ion }}$ and $F_{\text {id }}^{(e)}$ denote the ideal free energy of ions and electrons, and the last three terms represent an excess free energy arising from the electron-electron, ionion, and ion-electron interactions, respectively.

The pressure $P$, the internal energy $U$, and the entropy $S$ of an ensemble of fixed number of plasma particles in volume $V$ can be obtained using the thermodynamic relations $P=-(\partial F / \partial V)_{T}, \quad S=-(\partial F / \partial T)_{V}$, and $U=F+T S$. The second-order thermodynamic functions are derived by differentiating these first-order ones. The decomposition (2) induces the analogous decomposition of $P, U, S$, the heat capacity $C_{V}=(\partial S / \partial \ln T)_{V}$, and the logarithmic pressure derivatives $\chi_{T}=(\partial \ln P / \partial \ln T)_{V}$ and $\chi_{\rho}=-(\partial \ln P / \partial \ln V)_{T}$. Other second-order functions can be expressed through these by Maxwell relations (e.g., [1] ).

\section{A. Ideal electron-ion plasmas}

The free energy of a gas of $N_{j}=n_{j} V$ nonrelativistic classical ions of $j$ th kind is

$$
F_{\mathrm{id}}^{(j)}=N_{j} k_{B} T\left[\ln \left(n_{j} \lambda_{j}^{3} / g_{j}\right)-1\right],
$$

where $g_{j}$ is the spin multiplicity. The total free energy is given by the sum $F_{\text {id }}^{\text {ion }}=\sum_{j} F_{\text {id }}^{(j)}$. Analogous sums give $U, S, P$, and $C_{V}$. Since Eq. (3) contains $n_{j}$ under logarithm, these sums for $F$ and $S$ naturally include the entropy of mixing $S_{\text {mix }}=-k_{B} \sum_{j} N_{j} \ln x_{j}$.

The free energy of the electron gas is given by

$$
F_{\text {id }}^{(e)}=\mu_{e} N_{e}-P_{\text {id }}^{(e)} V,
$$

where $\mu_{e}$ is the electron chemical potential. The pressure $P_{\text {id }}^{(e)}$ and the number density $n_{e}=N_{e} / V$ are functions of $\mu_{e}$ and $T$, which can be written through the Fermi-Dirac integrals $I_{\nu}\left(\chi_{e}, \tau\right)$, where $\chi_{e}=\mu_{e} / k_{B} T$ and $\nu=1 / 2$, $3 / 2$, and $5 / 2$. In Ref. [1] we gave analytic approximations for the Fermi-Dirac integrals, based on fits [9] to electron-positron thermodynamic functions. The chemical potential at a given density can be found either from a numerical inversion of function $n_{e}\left(\chi_{e}, T\right)$ or using the analytic approximation [1]. The electron-gas contributions to $\chi_{T}, C_{V}$, and $S$ tend to zero at $T \ll T_{F}$. Related numerical problems and their cure will be discussed elsewhere 12.

\section{B. Nonideal plasmas containing one type of ions}

Let us recall fit formulae for nonideal EIP containing a single kind of ions.

a. Electron exchange and correlation. Electronelectron (exchange-correlation) effects were studied by many authors. For the reasons explained in Refs. [1, 12], we adopt the fit to $f_{e e} \equiv F_{e e} /\left(N_{e} k_{B} T\right)$ presented in Ref. [16].

b. One-component plasma. The internal energy of the liquid one-component plasma ( $\mathrm{OCP})$ at any values of $\Gamma$ is given by [2]

$$
u_{i i}=\Gamma^{3 / 2}\left(\frac{A_{1}}{\sqrt{A_{2}+\Gamma}}+\frac{A_{3}}{1+\Gamma}\right)+\frac{B_{1} \Gamma^{2}}{B_{2}+\Gamma}+\frac{B_{3} \Gamma^{2}}{B_{4}+\Gamma^{2}},
$$

where $u_{i i} \equiv U_{i i} / k_{B} T N_{\text {ion }}$, and

$$
A_{3}=-\sqrt{3} / 2-A_{1} / \sqrt{A_{2}}
$$

ensures the correct transition to the Debye-Hückel limit. The parameters $A_{1}=-0.907347, A_{2}=0.62849, B_{1}=$ $0.0045, B_{2}=170, B_{3}=-8.4 \times 10^{-5}$, and $B_{4}=0.0037$ allow one to reproduce the best available MC simulations of liquid OCP at $1 \leq \Gamma \leq 190$ [13] with an accuracy matching the numerical MC noise.

From Eq. (5) one obtains the analytic expression for $f_{i i} \equiv F_{i i} / k_{B} T N_{\text {ion }}$ by integration, and then the Coulomb contributions to the other thermodynamic functions by differentiation [2].

c. Electron polarization. Electron polarization in Coulomb liquid was studied by perturbation [14, 15] and HNC [1, 2, 4] techniques. The results for $f_{i e} \equiv$ $F_{i e} / N_{\text {ion }} k_{B} T$ have been fitted by the expression [2]

$$
f_{i e}=-\Gamma_{e} \frac{c_{\mathrm{DH}} \sqrt{\Gamma_{e}}+c_{\mathrm{TF}} a \Gamma_{e}^{\nu} g_{1}\left(r_{s}, \Gamma_{e}\right) g_{3}\left(x_{\mathrm{rel}}\right)}{1+\left[b \sqrt{\Gamma_{e}}+a g_{2}\left(r_{s}, \Gamma_{e}\right) \Gamma_{e}^{\nu} / r_{s}\right] \gamma_{r}^{-1}} .
$$

The coefficients $c_{\mathrm{DH}}, c_{\mathrm{TF}}, a, b, \nu$ and functions $g_{1,2}\left(r_{s}, \Gamma_{e}\right)$ and $g_{3}\left(x_{\mathrm{rel}}\right)$ parametrically depend on the ion charge $Z$. Here the coefficients $c_{\mathrm{DH}}$ and $c_{\mathrm{TF}}$ are not free fit parameters, because

$$
c_{\mathrm{DH}}=(Z / \sqrt{3})\left[(1+Z)^{3 / 2}-1-Z^{3 / 2}\right]
$$

ensures the transition of the excess free energy to the Debye-Hückel limit at small $\Gamma$, and $c_{\mathrm{TF}}$ at large $Z$ is given by the Thomas-Fermi theory [10]. 


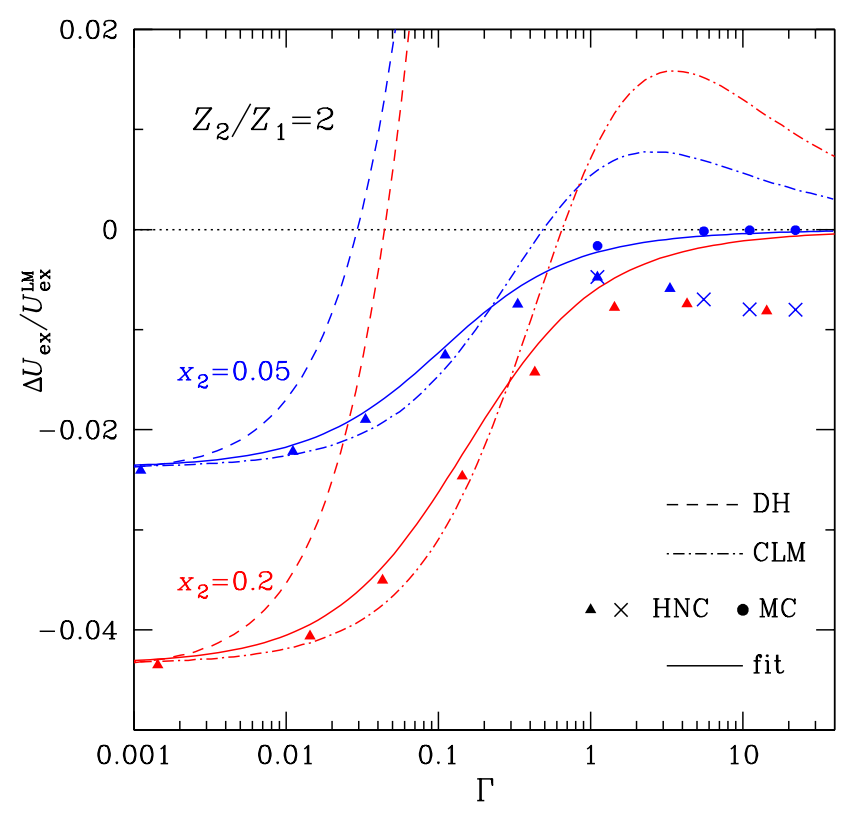

FIG. 1: Fractional difference between the Coulomb part of the internal energy $\left(U_{i i}\right)$ in different approximations and the LMR prediction as a function of the average ion Coulomb coupling parameter $\Gamma$ for binary mixtures of ions with $Z_{2} / Z_{1}=2$ and $x_{2}=1-x_{1}=0.05$ and 0.2 in the rigid background. Dashed lines (DH): Debye-Hückel formula, dot-dashed lines (CLM): corrected linear mixing [8]; dots: MC results of Ref. [6] for $x_{2}=0.05$; crosses: HNC results of Ref. [6]; triangles: present $\mathrm{HNC}$ results; solid lines: present fit.

\section{Nonideal mixtures of ions}

A common approximation for the excess (nonideal) free energy of the strongly coupled ion mixture is the LMR,

$$
f_{\mathrm{ex}}^{\mathrm{LM}}(\Gamma) \approx \sum_{j} x_{j} f_{\mathrm{ex}}\left(\Gamma_{j}, x_{j}=1\right)
$$

where superscript "LM" denotes the linear-mixing approximation, and all $\Gamma_{j}$ correspond to the same $\Gamma_{e}$ (assuming that the pressure is given almost totally by the strongly degenerate electrons): $\Gamma_{j}=\Gamma Z_{j}^{5 / 3} /\left\langle Z^{5 / 3}\right\rangle$. In Eq. (9), $f_{\text {ex }}$ is the reduced nonideal part of the free energy: $f_{\mathrm{ex}}=f_{i i}$ for the "rigid" (uniform) chargeneutralizing electron background and $f_{\text {ex }}=f_{i i}+f_{i e}+$ $Z_{j} f_{e e}$ for the polarizable background

The high accuracy of Eq. (9) for binary ionic mixtures in the rigid background was first demonstrated by calculations in the HNC approximation [3] and confirmed later by MC simulations (e.g., [5, 6, 6] ).

The validity of the LMR in the case of an ionic mixture immersed in a polarizable finite-temperature electron background has been examined by Hansen et al. 3] in the first-order thermodynamic perturbation approximation and by Chabrier and Ashcroft [4] by solving the HNC equations with effective screened potentials. These

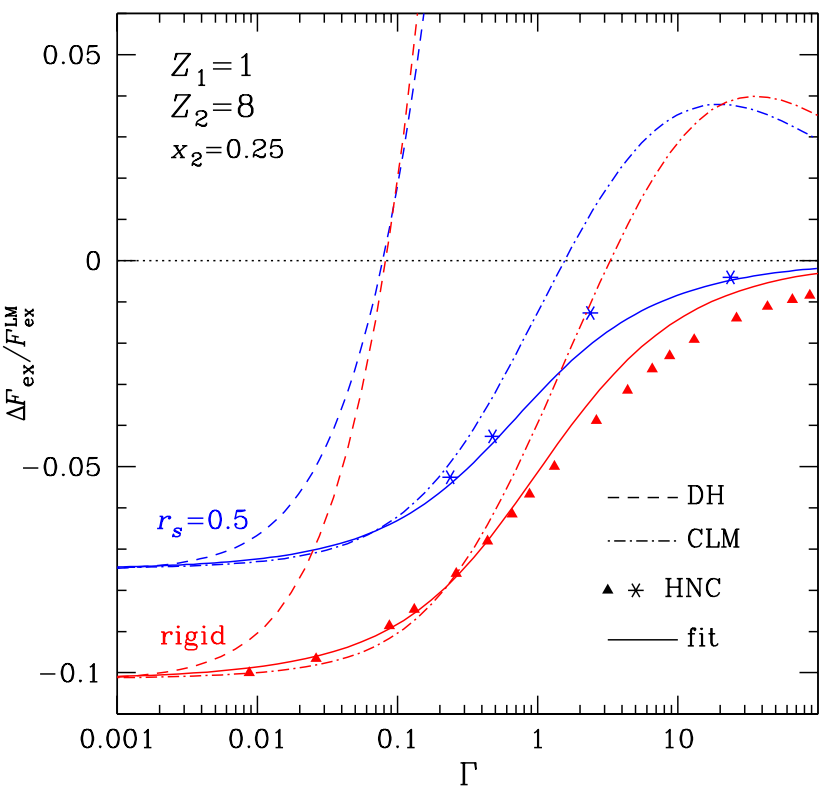

FIG. 2: Fractional differences between the nonideal part of the free energy due to the ion-ion and ion-electron interactions $\left(F_{i i}+F_{i e}\right)$ in different approximations and the LMR prediction for a mixture of ions with $Z_{1}=1$ and $Z_{2}=8$ and $x_{2}=$ $1-x_{1}=0.25$ in the rigid and polarizable electron background, as functions of $\Gamma$. Dashed lines: DH, dot-dashed lines: CLM [8]; asterisks: HNC results of Ref. [4]; solid lines: present fit.

authors found that the LMR remains accurate when the electron response is taken into account in the inter-ionic potential, as long as the Coulomb coupling is strong $(\Gamma>1)$.

On the other hand, the LMR is invalid at $\Gamma \ll 1$. Indeed, in this case the Debye-Hückel theory gives

$$
f_{e e}^{\mathrm{DH}}=-\Gamma_{e}^{3 / 2} / \sqrt{3}, \quad f_{i i}^{\mathrm{DH}}=f_{e e}^{\mathrm{DH}} \zeta_{i i}^{\mathrm{DH}},
$$

and for the EIP

$$
f_{\mathrm{ex}}^{\mathrm{DH}}=f_{i i}^{\mathrm{DH}}+f_{i e}^{\mathrm{DH}}+\langle Z\rangle f_{e e}^{\mathrm{DH}}=f_{e e}^{\mathrm{DH}} \zeta_{\text {eip }}^{\mathrm{DH}},
$$

where

$$
\zeta_{i i}^{\mathrm{DH}}=\frac{\left\langle Z^{2}\right\rangle^{3 / 2}}{\langle Z\rangle^{1 / 2}}, \quad \zeta_{\text {eip }}^{\mathrm{DH}}=\frac{\left(\left\langle Z^{2}\right\rangle+\langle Z\rangle\right)^{3 / 2}}{\langle Z\rangle^{1 / 2}} .
$$

However, the LMR at $\Gamma \ll 1$ gives another result:

$$
f_{i i}^{\mathrm{LM}} \sim f_{e e}^{\mathrm{DH}} \zeta_{i i}^{\mathrm{LM}}, \quad f_{\mathrm{ex}}^{\mathrm{LM}} \sim f_{e e}^{\mathrm{DH}} \zeta_{e i p}^{\mathrm{LM}},
$$

where

$$
\zeta_{i i}^{\mathrm{LM}}=\left\langle Z^{5 / 2}\right\rangle, \quad \zeta_{\text {eip }}^{\mathrm{LM}}=\left\langle Z(Z+1)^{3 / 2}\right\rangle .
$$

Nadyozhin and Yudin [8] considered several possible modifications of the LMR at intermediate $\Gamma$ and found that such modification can appreciably shift the statistical nuclear equilibrium at the conditions typical of the 


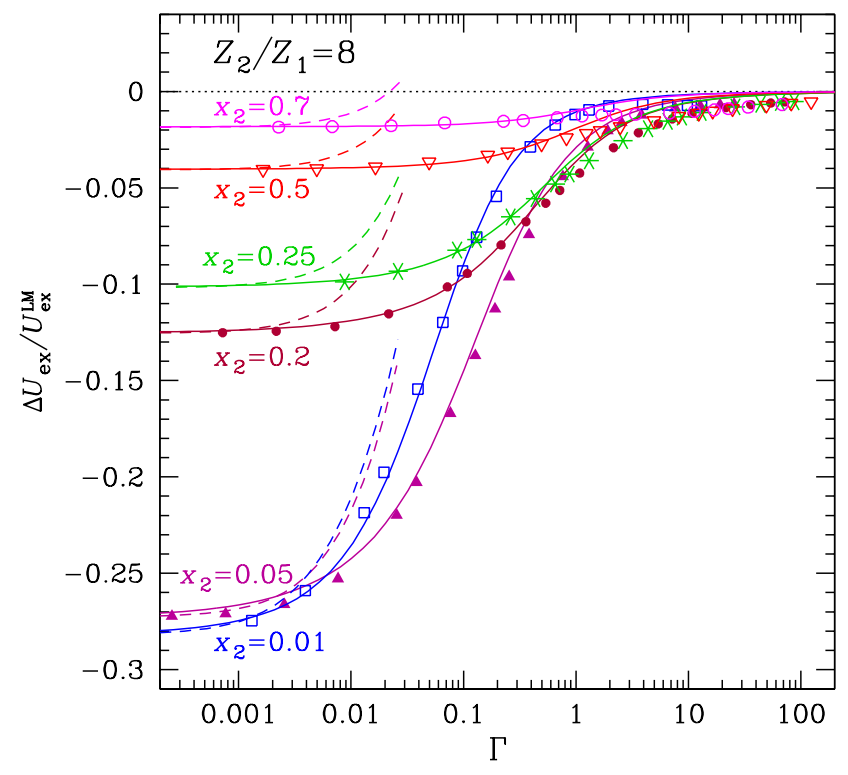

FIG. 3: Fractional difference between the Coulomb part of the internal energy in different approximations and the LMR prediction for a binary mixture of ions with $Z_{2} / Z_{1}=8$ in the rigid background. Dashed lines (DH): Debye-Hückel formula, symbols: present HNC results; solid lines: present fit. Different symbols correspond to different $x_{2}$ values: 0.01 (squares), 0.05 (solid triangles), 0.2 (dots), 0.25 (asterisks), 0.5 (empty triangles), and 0.7 (empty circles).

final stage of a stellar gravitational collapse. They considered the rigid background and advocated a modification of every term in Eq. (9) by multiplying the leading fit coefficient at small $\Gamma$ by factor $d_{j}=\sqrt{\left\langle Z^{2}\right\rangle / Z_{j}\langle Z\rangle}$. It corresponds to replacing $\sqrt{3} / 2$ in Eq. (6) by $d_{j} \sqrt{3} / 2$ (and a possible simultaneous change of $A_{2}$ ). For a compressible background an analogous modification implies additionally replacement of Eq. (8) by

$$
c_{\mathrm{DH}}^{(j)}=\frac{Z_{j}}{\sqrt{3}}\left[\left(\frac{\left\langle Z^{2}\right\rangle}{\langle Z\rangle}+1\right)^{3 / 2}-\frac{\left\langle Z^{2}\right\rangle^{3 / 2}}{\langle Z\rangle^{3 / 2}}-1\right] .
$$

Hereafter modifications of this type will be called corrected linear mixing (CLM).

The result of such modifications is shown by dotdashed lines in Figs. 11 and 2, where we plot the ratios $\Delta u_{i i} / u_{i i}^{\mathrm{LM}}$ and $\Delta f_{\mathrm{ex}} / f_{\mathrm{ex}}^{\mathrm{LM}}$ as functions of $\Gamma$. Here $\Delta f \equiv f-f^{\mathrm{LM}}$ and $\Delta u \equiv u-u^{\mathrm{LM}}$ are the deviations of the reduced free and internal energies, respectively, from the LMR. In Fig. 1 the electron response is neglected (rigid background). We see that, for example, at $\Gamma \approx 10$ the CLM prescription gives $\Delta u$ and $\Delta f$ corrections of about 1\% in Fig. 1 and several percents in Fig. 22, whereas they must be much smaller according to the $\mathrm{MC}$ results [6, 7]. Moreover, $\Delta u$ and $\Delta f$ in the CLM approximation have the incorrect sign at $\Gamma \gtrsim 1$ (note that according to

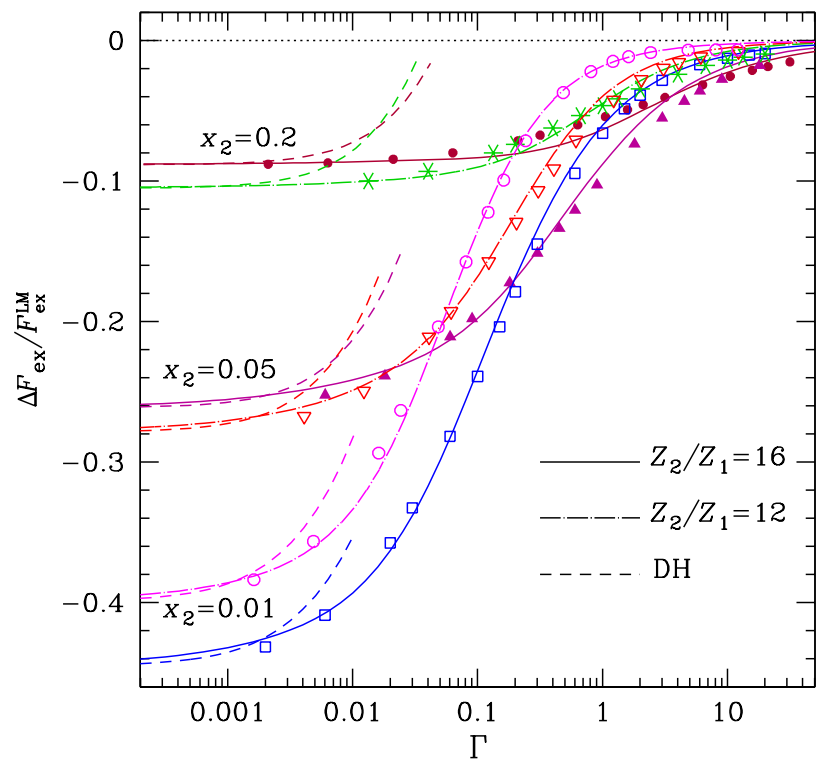

FIG. 4: Fractional difference between the Coulomb part of the free energy and the LMR prediction for binary ionic mixtures with $Z_{2} / Z_{1}=12$ and $Z_{2} / Z_{1}=16$, and with $x_{2}=0.01$, 0.05 , and 0.2. Dashed lines (DH): Debye-Hückel formula, symbols: present HNC results; solid lines: present fit for $Z_{2} / Z_{1}=16$; long-dash-dot lines: present fit for $Z_{2} / Z_{1}=12$. Different symbols correspond to different combinations of $x_{2}$ and $Z_{2} / Z_{1}$ values.

Ref. 3] $\Delta u / u$ and $\Delta f / f$ are negative at any $\Gamma)$. Additional modifications of the coefficient $A_{2}$ in Eq. (9) also do not solve the problem.

An alternative to the CLM, named "complex mixing" in Ref. 8], maintains the correct sign of $\Delta f$ and $\Delta u$, but leads to still larger absolute values of these corrections (i.e., still slower recovery of the LMR) at large $\Gamma$.

In order to find a more accurate approximation, we have performed HNC calculations of $F_{i i}, U_{i i}$ and $P_{i i}$ for binary ionic mixtures in the rigid background for a broad $\Gamma$ range and various values of the charge number ratios $Z_{2} / Z_{1}$ and fractional abundances $x_{2}=1-x_{1}$. Some of the results are shown by triangles in Figs. 1 and 2 . In agreement with the previous studies (e.g., [3]), our numerical results show monotonically decreasing fractional deviations from the LMR with increasing $\Gamma$. The results agree to at least 5 digits with those published in [6] (crosses in Fig. 11). At $\Gamma \gtrsim 1, \mathrm{HNC}$ results tend to a constant residual within $1 \%$, which is due to the intrinsic inaccuracy of the HNC approximation for strongly coupled plasmas because of the lack of the bridge functions in the diagrammatic representation of this approximation. To prove this statement, in Fig. 1 we plot by dots the values of $\Delta u_{i i} / u_{i i}^{\mathrm{LM}}$ from MC simulations [6]. The latter simulations give tiny deviations from the LMR at $\Gamma \gtrsim 3$, which are invisible in the figure scale. 


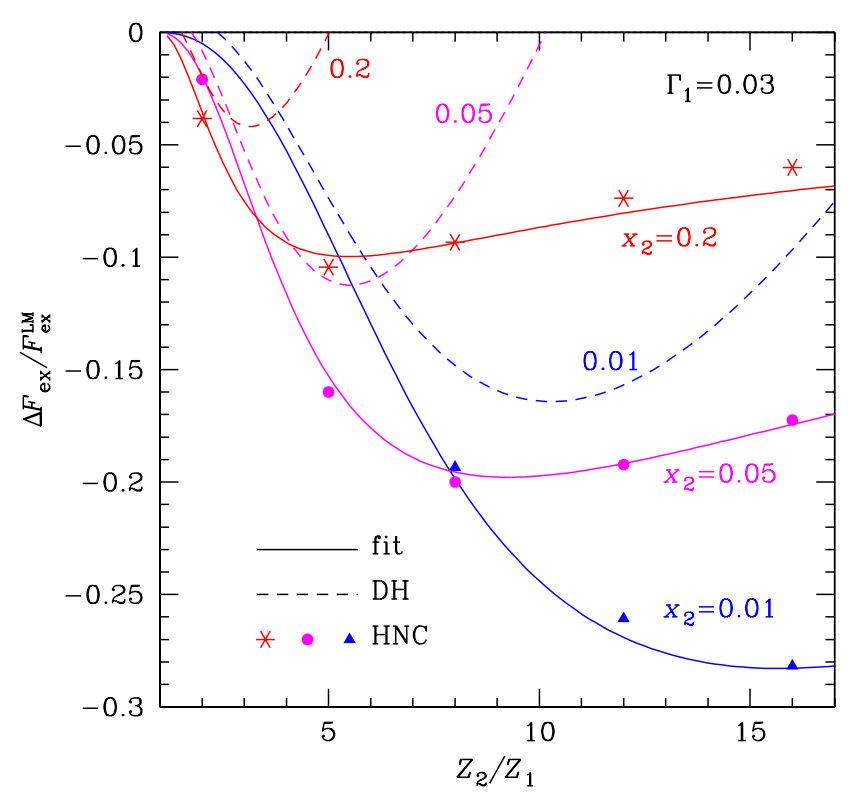

FIG. 5: Fractional difference between the Coulomb part of the internal energy and the LMR prediction for binary ionic mixtures at $\Gamma_{1}=0.03$ as function of the ion charge ratio $Z_{2} / Z_{1}$. Dashed lines (DH): Debye-Hückel formula, symbols: present HNC results; solid lines: present fit. Different symbols correspond to different $x_{2}$ values: $x_{2}=0.01$ (triangles), 0.05 (dots), and 0.2 (asterisks).

Asterisks in Fig. 2 correspond to the HNC calculations for mixtures in the polarizable electron background [4], which give qualitatively the same results as the calculations for the rigid background. The second dot-dashed curve in this figure shows CLM for the polarizable background, according to Eq. (15).

A correction to the linear mixing rule, which exactly recovers the Debye limit at $\Gamma \rightarrow 0$ and the LMR at $\Gamma \gg 1$, and which agrees with the HNC data, can be expressed by the following analytic fitting formula:

$$
\Delta f=f_{e e}^{\mathrm{DH}} \frac{\zeta^{\mathrm{DH}}-\zeta^{\mathrm{LM}}}{\left(1+a \Gamma^{b}\right)^{c}},
$$

where $\zeta=\zeta_{\text {ii }}$ or $\zeta_{\text {eip }}$ for the rigid or polarizable background, respectively, and the parameters $a, b$, and $c$ depend on plasma composition as follows:

$$
\begin{aligned}
& a=\frac{2.2 \delta+17 \delta^{4}}{1-b}, \quad \delta=\frac{\zeta^{\mathrm{LM}}-\zeta^{\mathrm{DH}}}{\left\langle Z^{5 / 2}\right\rangle} \\
& b=d^{-0.2}, \quad c=1+d / 6, \quad d=\left\langle Z^{2}\right\rangle /\langle Z\rangle^{2} .
\end{aligned}
$$

Thanks to the simple form of this formula, its derivatives are also rather simple. For example, the corrections to the reduced internal energy and heat capacity read,

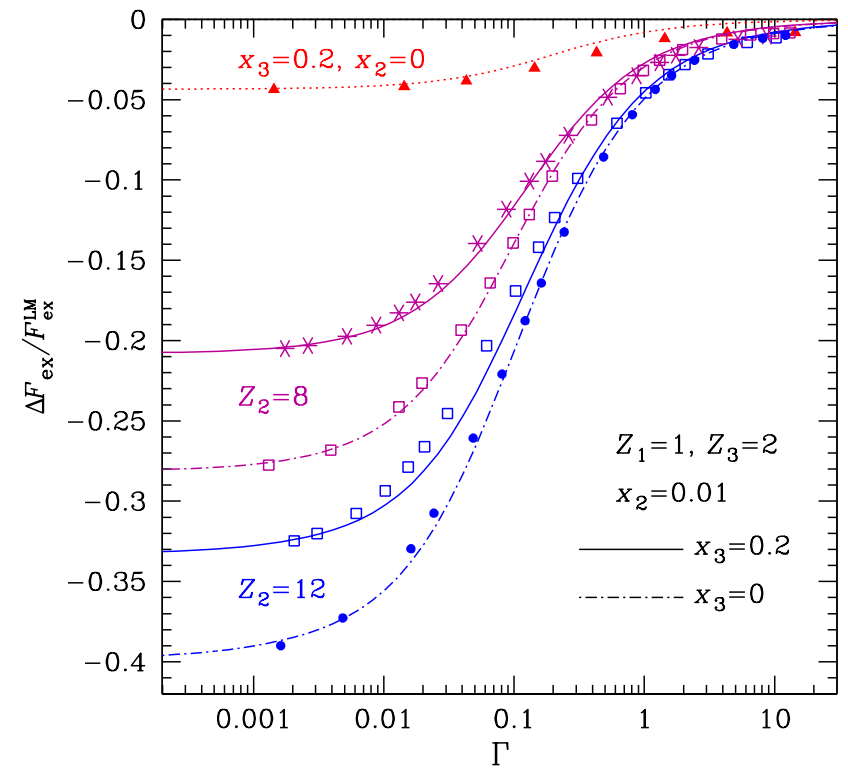

FIG. 6: Fractional difference between the Coulomb part of the free energy and the LMR prediction for mixtures of three (solid lines) and two (dot-dashed and dotted lines) types of ions with charge ratios $Z_{j} / Z_{1}=2,8$, and 12 . Different symbols correspond to HNC results with different combinations of $x_{j}$ and $Z_{j}$. Solid lines: $Z_{2} / Z_{1}=8$ or $12\left(Z_{2}\right.$ is marked near the curves, assuming $\left.Z_{1}=1\right), x_{2}=0.2 ; Z_{3} / Z_{1}=12$, $x_{3}=0.01$. Dot-dashed lines: the same but without the third kind of ions $\left(x_{3}=0\right)$. Dotted line: binary mixture with charge ratio $Z_{3} / Z_{1}=2\left(x_{2}=0\right)$ and number fraction $x_{3}=0.2$.

respectively,

$$
\begin{aligned}
\Delta u & =\left(\frac{3}{2}-\frac{a b c \Gamma^{b}}{1+a \Gamma^{b}}\right) \Delta f \\
\Delta c & =\left(\frac{a b c \Gamma^{b}}{1+a \Gamma^{b}}-\frac{1}{2}\right) \Delta u-\frac{a b^{2} c \Gamma^{b}}{\left(1+a \Gamma^{b}\right)^{2}} \Delta f .
\end{aligned}
$$

This approximation has been compared with our HNC calculations for binary ionic mixtures in the rigid electron background at $Z_{2} / Z_{1}=2,5,8,12$, and 16 , with $x_{2}=1-x_{1}$ ranging from 0.01 to 0.7 . Some of the results are shown in the figures. In all figures the Debye-Hückel approximation is drawn by dashed lines for comparison. In Figs. 1 and 2, discussed above, and in Figs. 3, 5 our approximation is drawn by solid lines. Figure 3 shows the fractional difference between the different results for internal energy and the LMR approximation for binary ionic mixtures with $Z_{2} / Z_{1}=8$ and $x_{2}=0.01,0.05,0.2$,

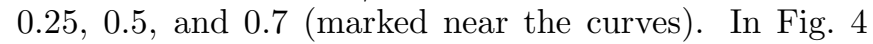
we show the case of the highest considered charge asymmetries: long-dash-dot lines show the approximation (16) for $Z_{2} / Z_{1}=12$ and solid lines for $Z_{2} / Z_{1}=16$. Finally, in Fig. 5 we show a dependence of $\Delta f_{i i} / f_{i i}^{\mathrm{LM}}$ on the charge ratio $Z_{2} / Z_{1}$ at $\Gamma_{1}=0.03$. Here different symbols correspond to the HNC results for different values of $x_{2}$. 


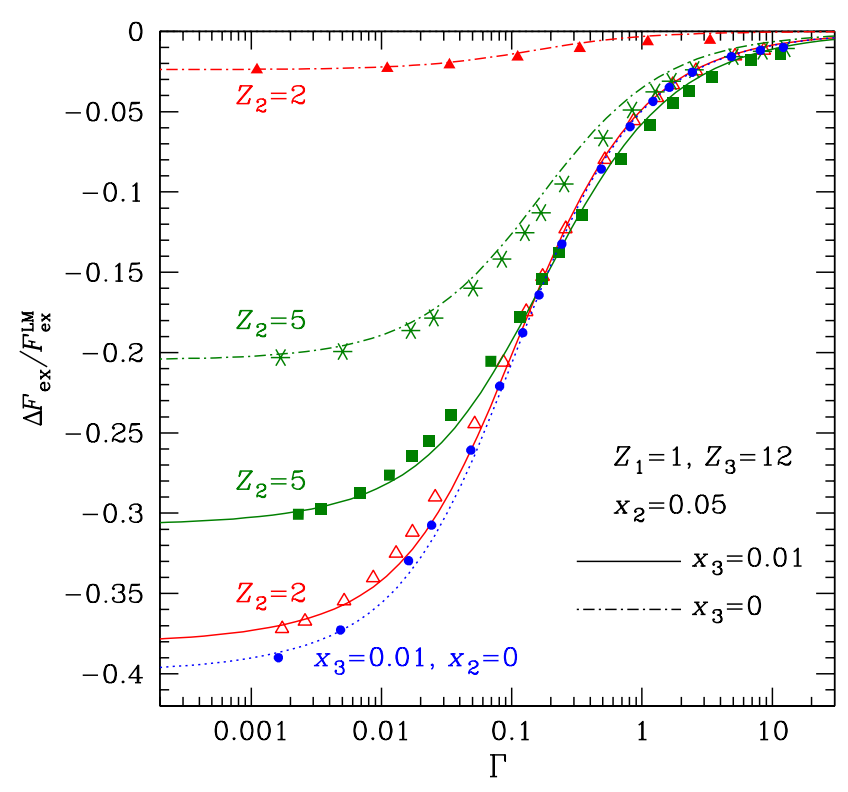

FIG. 7: Same as in Fig. 6] but for different charge ratios and fractional abundances. Solid lines: $Z_{2} / Z_{1}=2$ and 5 (marked near the curves), $x_{2}=0.05 ; Z_{3} / Z_{1}=12, x_{3}=0.01$. Dotdashed lines: the same but with $x_{3}=0$. Dotted line: the same with $x_{2}=0\left(x_{3}=0.01\right)$.

The difference between the HNC results and formula (16) for $f_{\mathrm{ex}}$ lies within 0.013 and within $1 \%$. The internal energy calculated using the analytic derivative of the fit (16) deviates from the HNC results by not more than 0.017 and not more than $1.5 \%$. These maximal deviations are attained for the extremely asymmetric mixtures with $Z_{2} / Z_{1}=16$.

The analytic formula (16) has also been compared to available HNC results for binary ionic mixtures in the polarizable electron background [4] and found to be satisfactory within the accuracy of the latter results (an example is shown in Fig. 2).
We have also performed calculations for mixtures of ions of three different types on the rigid electron background and compared the results with Eq. (16). The results of the comparison are shown in Figs. 6 and 7 . Solid lines show the difference of the Coulomb free energy from the LMR according to Eq. (16) for 3-component mixtures; for comparison, dot-dashed and dotted lines are plotted for 2-component mixtures with the same charge ratios; symbols represent the HNC results. In all considered cases, adding a third component to a binary mixture increases the deviations of Eq. (16) from HNC results by less than a factor of 1.5. We conclude that the agreement between the fit and numerical results remains satisfactory.

\section{CONCLUSIONS}

We have performed HNC calculations of the free energy, internal energy, and pressure for various ionic mixtures with different fractional abundances of the ion species in a broad range of $Z$ and $\Gamma$ values. We have constructed an analytic approximation to the deviation from the LMR, which recovers the Debye-Hückel formula for multicomponent plasmas at $\Gamma \ll 1$ and the LMR at $\Gamma \gg 1$, and which describes our calculations at any $\Gamma$ values, as well as the HNC and MC results for the internal energy of plasma mixtures, available in the literature, with an accuracy better than $2 \%$.

\section{Acknowledgments}

The work of G.C. and A.Y.P. was partially supported by the CNRS French-Russian Grant No. PICS 3202. The work of A.Y.P. was partially supported by the Rosnauka Grant NSh-2600.2008.2 and the RFBR Grant 0802-00837. The work of F.J.R. was partially performed under the auspices of the U.S. Department of Energy by Lawrence Livermore National Laboratory under Contract DE-AC52-07NA27344.
[1] G. Chabrier and A. Y. Potekhin, Phys. Rev. E 58, 4941 (1998).

[2] A. Y. Potekhin and G. Chabrier, Phys. Rev. E 62, 8554 (2000).

[3] J. P. Hansen, G. M. Torrie, and P. Vieillefosse, Phys. Rev. A 16, 2153 (1977).

[4] G. Chabrier and N. W. Ashcroft, Phys. Rev. A 42, 2284 (1990).

[5] Y. Rosenfeld, Phys. Rev. E, 52, 3292 (1995); 54, 2827 (1996).

[6] H. DeWitt, W. Slattery, and G. Chabrier, Physica B 228, 21 (1996).

[7] H. E. DeWitt and W. Slattery, Contrib. Plasma Phys. 43, 279 (2003).

[8] D. K. Nadyozhin and A. V. Yudin, Astronomy Letters
31, 271 (2005).

[9] S. I. Blinnikov, N. V. Dunina-Barkovskaya, and D. K. Nadyozhin, Astrophys. J. Suppl. Ser. 106171 (1996); 118, 603(E) (1998).

[10] E. E. Salpeter, Astrophys. J. 134, 669 (1961).

[11] L. D. Landau and E. M. Lifshitz, Statistical Physics, Part 1 (Pergamon, Oxford, 1993).

[12] A. Y. Potekhin and G. Chabrier (unpublished).

[13] J. M. Caillol, J. Chem. Phys. 111, 6538 (1999).

[14] S. Galam and J. P. Hansen, Phys. Rev. A 14, 816 (1976).

[15] D. G. Yakovlev and D. A. Shalybkov, Sov. Astron. Lett. 13, 308 (1987); Sov. Sci. Rev. E: Astrophys. Space Phys. Rev. 7, 311 (1989).

[16] S. Ichimaru, H. Iyetomi, and S. Tanaka, Phys. Rep. 149, 91 (1987). 